\title{
The when and where of spatial storage in memory-guided saccades
}

\author{
Debora Brignani $^{\text {a }}$, Marta Bortoletto ${ }^{\text {a }}$, Carlo Miniussi ${ }^{\mathrm{a}, \mathrm{b}, *}$, Claudio Maioli $^{\mathrm{b}}$ \\ a Cognitive Neuroscience Section, IRCCS San Giovanni di Dio Fatebenefratelli, Brescia, Italy \\ b Department of Biomedical Sciences and Biotechnology, National Institute of Neuroscience, University of Brescia, Italy
}

\section{A R T I C L E I N F O}

\section{Article history:}

Received 22 October 2009

Revised 12 February 2010

Accepted 13 May 2010

Available online 21 May 2010

\section{Keywords:}

memory-guided saccades

parietal cortex

frontal eye fields

spatial working memory

ERP

SLORETA

\begin{abstract}
A B S T R A C T
The memory-guided saccade paradigm is an ideal experimental model for studying spatial working memory. Both the posterior parietal cortex and frontal cortex are known to play a role in working memory; however, there is much debate about the degree of their involvement in the retention of information. We used eventrelated potentials and electromagnetic tomography to clarify the precise time course and location of the neural correlates of spatial working memory during a memory-guided saccade task in humans. We observed sustained activity in the inferior parietal lobe and extrastriate areas that persisted for the entire duration of the sensory- and memory-phases. This time course reveals that these regions participate in both initial sensory processing of visual cues and in the short-term maintenance of spatial location memory. Similar sustained activation was also observed in the anterior cingulate cortex, probably reflecting attentive control during the task. Differential activity between conditions was also recorded in the dorsolateral prefrontal cortex and in the frontal eye fields, but only during the initial part of the memory-phase. This finding suggests that these areas are not involved in the storage of spatial information, but rather in response selection and in transformation of spatial information into a motor coordinate framework, respectively. By exploiting techniques that provide exquisite temporal resolution and reasonably precise anatomical localization, this study provides evidence supporting the key role of inferior parietal lobe in the storage of spatial information during a working memory guided saccade.
\end{abstract}

(c) 2010 Elsevier Inc. All rights reserved.

\section{Introduction}

Spatial working memory (WM) refers to the ability to temporary retain spatial information that is not currently accessible in the perceptual domain and to use that information to effectively interact with the external environment (Baddeley, 1986; D'Esposito, 2007). The saccadic oculomotor system is an ideal experimental model for the study of spatial WM, since it permits to isolate the activity related to the maintenance of information from that related to the visual processing of the cue and to the response execution.

Generally, in a memory-guided saccade (MS) paradigm (Hikosaka and Wurtz, 1983), subjects remember the location of a briefly flashed visual cue and perform a saccade toward it at the end of a delay interval. Cortical areas showing persistent activity throughout the duration of the memory interval are thought to be directly involved in the active maintenance of the information hold in WM (Curtis and D'Esposito, 2003). During the retention of spatial information, a broad range of frontal-parietal cortical areas exhibit persistent neural activity (D'Esposito, 2007; Postle, 2006). Their involvement in the active maintenance of information, however, depends on the type

\footnotetext{
* Corresponding author. Department of Biomedical Sciences and Biotechnologies, University of Brescia, Viale Europa 11, 25123 Brescia, Italy.

E-mail address: miniussi@med.unibs.it (C. Miniussi).
}

of information to be remembered and on the response modality required in the task. In an MS paradigm, it is thought that frontal eye fields (FEFs) are largely involved when subjects are biased toward the use of a prospective motor code, such as in the maintenance of the motor plan as oculomotor coordinates (Curtis et al., 2004; Fuster, 1995; Postle and D'Esposito, 2003). Conversely, a greater contribution of parietal cortex is expected when subjects maintain a retrospective perceptual code, in which attention is allocated to regions of the extrastriate and parietal cortex responsible for the perception of location (Awh and Jonides, 2001; Curtis et al., 2004; Postle et al., 2004). However, a recent study has provided evidence that is inconsistent with these assumptions, raising doubts concerning the nature of the code carried by the persistent delay period activity (Srimal and Curtis, 2008). These authors (Srimal and Curtis, 2008) used event-related functional magnetic resonance imaging to identify cortical areas with a persistent activity throughout the WM delays and tested if this activity was related to the metrics of the MS response. They found that during the delay period, activity in the FEFs did not differ from activity in the parietal area, even when saccades were never made, challenging therefore the assumption that neurons in the FEFs maintain the metrics for saccades.

In the present study, we exploit the high temporal resolution of event-related potentials (ERPs) to understand the mechanisms of maintenance during spatial WM delays in humans. Indeed, an important step toward revealing the specific contribution of the 
frontal-parietal areas may come from determining the relative timing of their sustained activation. To our knowledge, two other studies investigated ERPs during memory-guided saccade paradigm (Evdodokimidis et al., 2001; Rama et al., 1995), but they did not address this issue of the nature of the code carried by the persistent delay period activity nor tried to localize the cortical areas involved.

A substantial difficulty in interpreting neural differences between a memory-guided saccade and a control condition is that there are numerous processes that occur while a subject is performing a simple memory task that have little or nothing to do with WM per se. These non-specific, task-general processes (e.g., arousal, sustained attention, general response preparation, etc.) may have directly contributed to the contradictory results reported in literature. In addition, the timing of neural modulation can be influenced by the specific intervals used between stimuli and the temporal expectations that these engender (Nobre et al., 2007). In the study of ERPs, then, also the least physical differences between the perceptual attributes of cueing stimuli may have a large impact on the ERP waveform morphology (Hillyard and Picton, 1979).

Keeping these aspects in mind, we compared ERPs elicited by an MS and a visually guided saccade (VS) task (e.g., Brignani et al., 2007). Both the tasks were built in order to minimize cognitive and perceptual differences unrelated to spatial working memory. Perceptual attributes of cueing stimuli, covert attentional shift and aspecific motor activation were carefully balanced in the two task conditions, all aspects that have not been considered before. In the MS task, subjects had to memorize the specific location of a saccadic target briefly presented during the sensory phase and perform an eye movement toward it at the end of the memory phase. In the VS task, subjects did not know the amplitude of the saccade to be executed, and simply moved toward a visually presented saccadic target at the end of the delay interval. However, also in this condition the direction of the subsequent saccade was provided, forcing subjects to shift spatial attention and to prepare a general motor plan towards the side where the saccadic target would have appeared. If FEFs play a relevant role in the storage of spatial information by maintaining the saccadic plan when the specific oculomotor coordinates are provided before the memory delay, then we expected a larger sustained activity of FEFs in MS than in VS task condition during all the delay period. On the contrary, if no persistent differential activation between the two tasks was found, we should conclude that FEFs do not play a relevant role in the storage of spatial information.

Despite excellent temporal resolution, ERPs lack the spatial discriminatory power to provide an adequate localization of the cortical activity. To overcome this difficulty we also analyzed data using standardized low resolution electromagnetic tomography (sLORETA, Pascual-Marqui, 2002, 2007), a method with zero error localization that seems in some aspects comparable to the classical functional imaging methods, when the analysis is limited to the identification of superficial signal sources within the cerebral cortex.

\section{Materials and methods}

\section{Participants}

Twenty-four healthy volunteers participated in the study. Four were disqualified from the analysis due to excessive noise in the recording, or because they produced too many anticipatory eye movements. The remaining 20 participants ( 8 females and 12 males) had a mean age of 23.5 years (range of 20-30). They were right handed $(+78.3 \%)$ according to the Edinburgh handedness inventory test (Oldfield, 1971) and had normal or corrected to normal visual acuity. The experimental methods were non-invasive and were approved by the Ethics Committee of IRCCS San Giovanni di Dio Fatebenefratelli, Brescia, Italy. All participants gave informed consent.

\section{Behavioral task and procedure}

Each participant sat in a dimly illuminated room with his/her head stabilized through a combination of a chin rest and a head support bar. All visual stimuli were rear-projected on a large screen placed at a distance of $1.5 \mathrm{~m}$ in front of the subject.

All participants performed an MS and a VS task, arranged in a blocked design and presented in a balanced order across subjects. In the MS task, subjects were required to memorize the position of a peripherally flashed visual target and to execute a saccade towards its position after a delay-phase. In the VS task, subjects did not need to memorize a saccadic target. After a delay interval, in which they were requested to maintain central fixation, they performed a saccade towards a peripheral target presented in the visual hemifield. In this task, information about the direction of the subsequent saccade was also provided, forcing subjects to shift spatial attention and to prepare a general motor plan towards the side where the saccadic target would have appeared.

The tasks are described in greater detail below. Several phases were common to both tasks, and all phases were presented in the same temporal sequence (Fig. 1):

Fixation-phase-Participants fixated on a central point for a period of $2 \mathrm{~s}$.

Sensory-phase-Two horizontal lines were shown for $300 \mathrm{~ms}$ on both sides of the central fixation point at $\pm 20^{\circ}$ of the visual angle, one with the same color as the central fixation point (i.e., cue), the other with a different color (i.e., distracter). In the VS task, the cueing horizontal line informed participants about the direction of the subsequent saccade (i.e., the hemifield containing the line of the same color as the central fixation point), inducing activation of a general motor plan. In the MS task, a dot with a color congruent to the line was superimposed on each horizontal line in one of eight possible positions, equally spaced along the horizontal meridian, within a range of $\pm 20^{\circ}$ of the visual field (i.e., $\pm 5^{\circ}$, $\left.\pm 10^{\circ}, \pm 15^{\circ}, \pm 20^{\circ}\right)$. The point with the same color as the central fixation point (i.e., the cue) was to be memorized, while the point appearing in a non-symmetrical position (i.e., a distracter) in the contralateral visual field was to be ignored. In this condition, the actual amplitude of the saccadic response was provided, allowing participants to program a specific motor plan. The horizontal lines and the distracter were included to reduce the visual differences between the two conditions and the two hemispheres, respectively.

Memory/delay-phase-The central fixation point was shown alone for a delay period of $2 \mathrm{~s}$, during which the subjects were instructed to avoid making eye movements.

Motor-phase-In the MS task, disappearance of the fixation point (i.e., GO signal) was the indication for subjects to execute a saccade to the precise remembered cue position (multiple saccades were allowed). In the VS task, disappearance of the fixation dot was followed by a $300 \mathrm{~ms}$ gap, after which a peripheral target (i.e., GO signal) appeared on the same side cued in the sensory-phase. In this task, subjects executed a simple saccade to the new target.

Correction-phase-This phase was present only during the MS task. One second after the GO signal, the memorized target appeared alone for $1 \mathrm{~s}$ in order to allow subjects to perform a corrective saccade if needed and additionally to check for possible errors in the response.

In both tasks, reappearance of the fixation point marked the beginning of the next trial.

The central fixation and the peripheral stimuli were red or green dots with a diameter of $0.43^{\circ}$, rendered on a black background. For half of the subjects, the central fixation point and, therefore, the valid cue were red, while, for the other half of the subjects, they were green. Each MS/VS experimental session was divided into four blocks of 64 


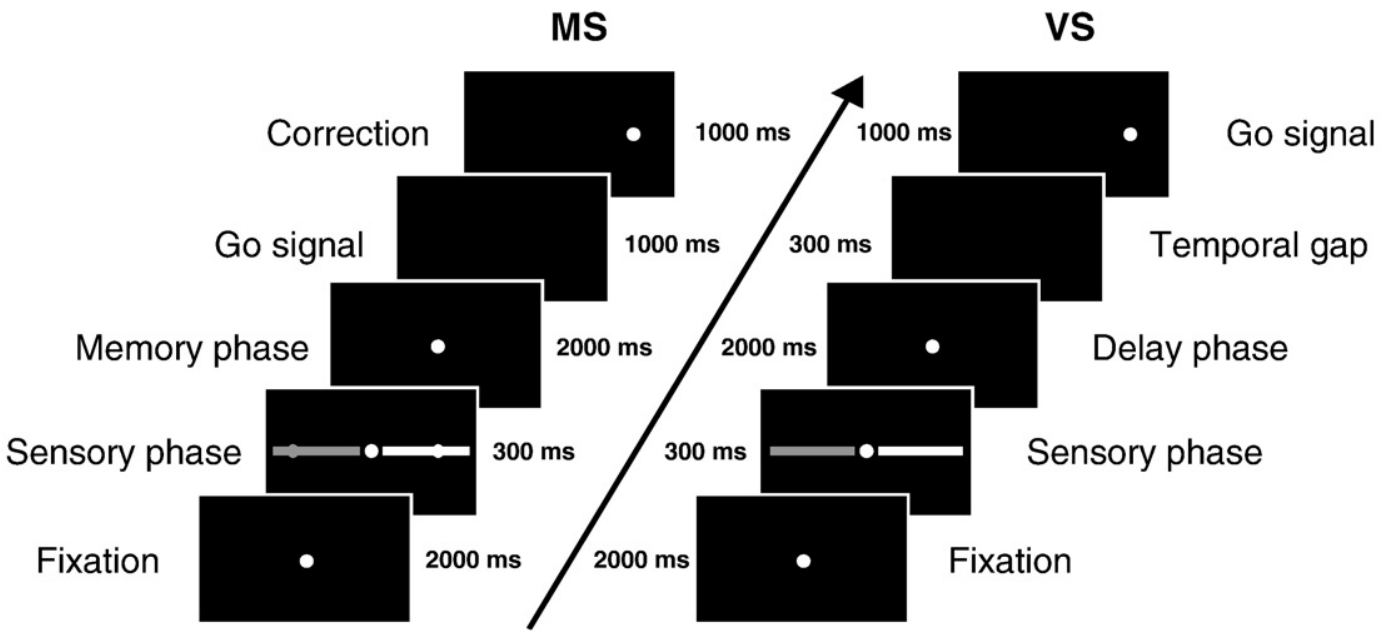

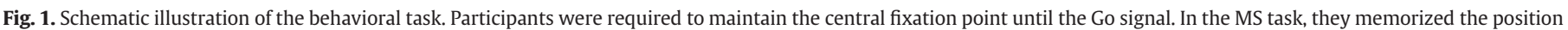

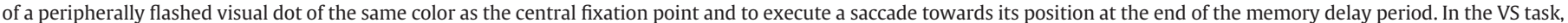

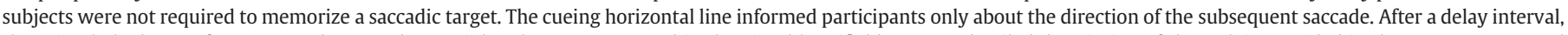

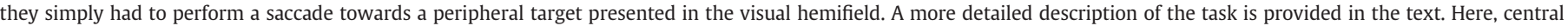
fixation point and visual cues are depicted in white and gray, respectively; however, they were presented in red and green, respectively, during the experiment.

trials (total number of trials: 256 for MS session and 256 for VS session). In all, each of the eight potential target locations $( \pm 5, \pm 10$, \pm 15 or $\pm 20^{\circ}$ of eccentricity) was used 32 times in a random order. At the beginning of the experiment, participants were informed about the relationship between the colors and the cue/distracter; this relationship was kept constant across the task conditions. Before each session, a training block familiarized subjects with the task condition. The whole experiment lasted for about $2 \mathrm{~h}$, including short rest breaks after each block of trials. To ensure clean ERP recordings, participants were requested to minimize blinking and to relax their muscles while performing the tasks.

\section{Behavioral analysis}

The EOG signal was visually inspected for each trial to evaluate the participants' performance. We determined saccade direction and latency (the interval between the GO signal and the saccade onset). Trials with blinks or saccades during the sensory or memory/delayphases were excluded from the analysis, as well as trials with anticipatory (latency $<140 \mathrm{~ms}$ ) or delayed (latency $>1000 \mathrm{~ms}$ ) eye movements or saccades in the wrong direction.

The accuracy of saccadic movements was calculated as absolute error, corresponding to the absolute value of the difference (in degrees) between the amplitude of the performed saccade and the required target position. Statistical analysis was carried out using repeated-measures analysis of variance (ANOVA), testing the task condition (MS, VS) and the memorized/cued side (left, right) as factors.

\section{Recordings and data processing}

Electroencephalographic (EEG) activity was recorded continuously from 29 sites using tin electrodes set in an elastic cap (Electro-Cap International, Inc.) and positioned according to the 10-20 international system (AEEGS, 1991). The montage included 3 midline sites ( Fz, Cz, Pz), 13 sites over each hemisphere (FP1/FP2, F3/F4, F7/F8, FC1/FC2, FC5/FC6, C3/C4, T7/T8, CP1/CP2, CP5/CP6, P3/P4, P7/P8, $\mathrm{PO} 7 / \mathrm{PO} 8,01 / \mathrm{O} 2)$ and the right mastoid M2. Additional electrodes were used as ground (i.e., electrode placed in front of $\mathrm{Fz}$ ) and the left mastoid M1 as reference site. Data were recorded with a band-pass filter of DC-100 Hz and digitized at a sampling rate of $500 \mathrm{~Hz}$ (SynAmps, NeuroScan). Electrodes-skin impedance was set below $5 \mathrm{k} \Omega$.
The continuous EEG recording was segmented offline, after it had been re-referenced to a weighted mean of both mastoid electrodes (M1, M2) and re-filtered digitally with a band-pass of $.01-60 \mathrm{~Hz}$. Epochs synchronized with the cue onset started $200 \mathrm{~ms}$ before the cue presentation and ended at the offset of the central fixation point $(+2300 \mathrm{~ms})$. All amplitude values were referred to the $200-\mathrm{ms}$ precue baseline. Trials with eye blinks, incorrect saccadic responses or muscle artifacts were excluded from analysis. In addition, trials were automatically eliminated if the voltage exceeded $\pm 100 \mu \mathrm{V}$ at FP1, FP2, $\mathrm{Fz}, \mathrm{Cz}, \mathrm{Pz}$ channels. In total a mean of $78( \pm 17)$ and $82( \pm 22)$ trials were rejected for each subject, respectively in MS and VS task conditions. The remaining trials were used to compute separate averages for each task condition (MS, VS) and for each cued direction (left, right).

Horizontal and vertical eye movements were recorded (DC-200 Hz low-pass filtered) by means of electrooculography (EOG). $\mathrm{Ag}-\mathrm{AgCl}$ electrodes were placed at the external canthi and above and below the right eye. EOG calibration was repeated before each experimental block and drift of DC offset was compensated within each trial by making the subject to look at a central fixation target before stimulus presentation. Oculomotor behavior was checked online on a monitor to assess the correctness of task execution, and recorded at a sampling rate of $500 \mathrm{~Hz}$ for subsequent offline analysis. Verbal feedback was given to subjects during the short breaks through the experimental sessions.

\section{ERP analysis}

Cue-related ERPs were analyzed in a systematic way to reveal differences between task conditions. Six different time-windows were individualized according to the visual inspection of the ERP components and to the results of an exploratory analysis of the data performed across the entire epoch (i.e., 0-2300 ms) by means of statistical cluster plots (see Guthrie and Buchwald, 1991; Murray et al., 2002): (a) the characteristic visual potential N1 (160-180 ms), in four pairs of parietal-occipital electrodes (P3/4, P7/8, PO7/8, 01/ 2); (b) a sustained frontal-polar difference between 200 and $2300 \mathrm{~ms}$ on FP1/2; (c) a central-parietal difference between 250 and $400 \mathrm{~ms}$ in five pairs of electrodes (FC1/2, C3/4, CP1/2, CP5/6, P3/4); (d) a frontal-temporal difference between 500 and $1000 \mathrm{~ms}$ in three pairs of electrodes (F7/8, T7/8, FC5/6); (e) a parietal contralateral delay activity (CDA) between 800 and $2300 \mathrm{~ms}$ in three pairs of parietal 
Table 1

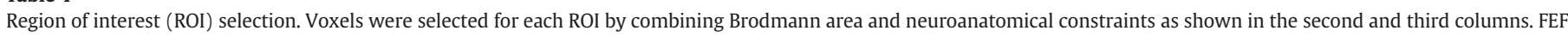

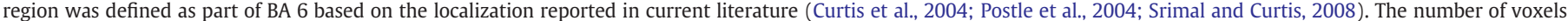

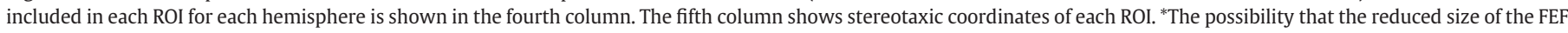
ROI compared with the other ROIs could have been a confound for results was excluded performing a control analysis on a larger ROI.

\begin{tabular}{|c|c|c|c|c|}
\hline Region of interest & Brodmann areas constraint & Neuroanatomical constraint & Voxels in contra-/ipsilateral hemisphere & Stereotaxic coordinates \\
\hline ACC & $24-32-33$ & $\begin{array}{l}\text { Anterior cingulate cortex } \\
\text { Cingulate gyrus }\end{array}$ & $159 / 145$ & $\begin{array}{l}x: 0 \text { to } \pm 20 \\
y: 50 \text { to }-25 \\
z:-10 \text { to } 50\end{array}$ \\
\hline dIPFC & $9-46$ & $\begin{array}{l}\text { Superior frontal gyrus } \\
\text { Middle frontal gyrus } \\
\text { Inferior frontal gyrus }\end{array}$ & $120 / 103$ & $\begin{array}{l}x: \pm 5 \text { to } \pm 60 \\
y: 55 \text { to } 0 \\
z: 5 \text { to } 40\end{array}$ \\
\hline FEF & 6 & $\begin{array}{l}\text { Middle frontal gyrus } \\
\text { Precentral gyrus }\end{array}$ & $27 / 22^{*}$ & $\begin{array}{l}x: \pm 45 \text { to } \pm 65 \\
y: 15 \text { to }-5 \\
z: 40 \text { to } 60\end{array}$ \\
\hline IPL & 40 & Inferior parietal lobule & $137 / 140$ & $\begin{array}{l}x: \pm 35 \text { to } \pm 65 \\
y:-25 \text { to }-65 \\
z: 20 \text { to } 60\end{array}$ \\
\hline ESA & $18-19$ & $\begin{array}{l}\text { Superior occipital gyrus } \\
\text { Middle occipital gyrus } \\
\text { Inferior occipital gyrus } \\
\text { Cuneus; lingual gyrus }\end{array}$ & $249 / 280$ & $\begin{array}{l}x: 0 \text { to } \pm 55 \\
y:-45 \text { to }-100 \\
z:-20 \text { to } 35\end{array}$ \\
\hline
\end{tabular}

electrodes (P3/4, P7/8, PO7/8); (f) a central CDA between 1000 and $2300 \mathrm{~ms}$ in three pairs of central electrodes (FC1/2, C3/4, CP1/2).

The mean amplitude within each temporal interval was analyzed separately through repeated-measures ANOVAs, by testing for three factors: task condition (MS, VS), hemisphere (ipsilateral, contralateral relative to the memorized/cued side) and electrode site. The Greenhouse-Geisser epsilon correction factor was applied where appropriate to compensate for possible effects of non-sphericity in the measurements. To clarify the pattern of significant interactions between experimental factors, selected two-sample comparisons were performed by means of $t$-tests for paired data with Bonferroni correction.

\section{Cortical localization analysis}

Despite excellent temporal resolution, ERPs lack the spatial discriminatory power to provide an adequate localization of cortical activity. To overcome this difficulty, we analyzed data using sLORETA (sLORETA, Pascual-Marqui, 2002, 2007). SLORETA (Pascual-Marqui, 2002, 2007) is a distributed underdetermined imaging method for localization of cerebral sources of EEG. The solution space consists of 6239 voxels ( $5 \mathrm{~mm}$ resolution) and matches the cortical gray matter volume. The electric potential lead field is computed by applying the boundary element method to the Montreal Neurological Institute 152 (MNI152) template (Fuchs et al., 2002). The sLORETA method estimates the current density distribution consistent with the scalp topography using the minimum norm least squares analysis, and weighs the solution with estimated electric potential variance. The result is a blurred widespread solution. The localization of sources is expressed in the MNI stereotaxic coordinates associated with quantitative neuroanatomy produced by the Talairach Daemon (Lancaster et al., 2000).

In the present study, sLORETA was used to localize cortical activity associated with the MS and VS tasks, and to characterize its time course. Average waveforms were re-referenced to the mean of all electrodes (average reference) and the left and right mastoids were removed from the dataset so that only 29 electrodes remained (see Recordings and data processing section).

We compared sLORETA images between MS and VS task conditions in five a priori regions of interest (ROI) motivated by past studies of spatial WM (Curtis et al., 2004; Postle et al., 2004; Srimal and Curtis, 2008). In sLORETA, voxels are defined with both cytoarchitectonic (i.e., Brodmann area) and neuroanatomic labels based on the Talairach Daemon atlas (Lancaster et al., 2000). We defined four
ROIs by choosing the voxels according to these two criteria. These included the anterior cingulate cortex (ACC), the dorsolateral prefrontal cortex (dIPFC), the inferior parietal lobule, (IPL) and the extrastriate areas (ESA).

The ACC (159 voxels in the contralateral and 145 voxels in the ipsilateral hemisphere) covered a region extending from $x: 0$ to \pm 20 , $y:-25$ to $50, z:-10$ to 50 and included voxels from Brodmann areas 24, 32 and 33, corresponding to the anterior cingulate cortex and the cingulate gyrus. The dIPFC (120 voxels in the contralateral and 103 voxels in the ipsilateral hemisphere) covered a region extending from $x$ : \pm 5 to $\pm 60, y$ : 0 to $55, z$ : 5 to 40 and included voxels from Brodmann areas 46 and 9, corresponding to the superior, middle and inferior frontal gyrus. The IPL (137 voxels in the contralateral and 140 voxels in the ipsilateral hemisphere) covered a region extending from $x: \pm 35$ to $\pm 65, y:-25$ to $-65, z: 20$ to 60 and included voxels from Brodmann areas 40 , corresponding to the inferior parietal lobule. The ESA ( 249 voxels in the contralateral and 280 voxels in the ipsilateral hemisphere) covered a region extending from $x$ : 0 to $\pm 55, y$ : -45 to $-100, z$ : -20 to 35 and included voxels from Brodmann areas 18 and 19 , corresponding to the superior, middle and inferior occipital gyrus, the cuneus and lingual gyrus.

In addition, an ROI corresponding to the FEFs was defined as part of BA 6 according to the localization reported in previous studies (Curtis et al., 2004; Paus, 1996; Postle et al., 2004). The FEF (27 voxels in the contralateral and 22 voxels in the ipsilateral hemisphere) covered a region extending from $x$ : \pm 45 to $\pm 65, y$ : 15 to $-5, z$ : 40 to 60 and corresponded to the middle frontal and precentral gyrus. All the details about the definition of each ROI are presented in Table 1.

The mean standardized current density value of each ROI was computed for each task condition and for each time point of the whole epoch (from 0 to $2300 \mathrm{~ms}$ after cue presentation; 1150 points), in the ipsi- and contra-lateral hemispheres separately. ${ }^{1}$ For each ROI, a twoway repeated measures ANOVA was carried out at each time point, testing for the task condition (MS, VS) and hemisphere factors (ipsilateral, contralateral relative to the memorized/cued side). Differences were considered significant only when $p$ values were less than 0.05 . Because of the multiple interrelated comparisons, and hence the likelihood of false-positive spurious significances, results

\footnotetext{
${ }^{1}$ In order to avoid potential confounds related to the different number of voxels included in the two hemispheres, we performed the analysis twice. In one analysis, we considered the left $=$ ipsilateral hemisphere and the right $=$ contralateral hemisphere; in the second analysis, we considered right $=$ ipsilateral and left $=$ contralateral. Since the two analyses produced the same results, only the outcomes of the former are reported.
} 
were only considered reliable if they persisted for more than 10 consecutive time bins ( 10 points equal to $20 \mathrm{~ms}$ ).

\section{Results}

\section{Behavioral data}

Analysis of the accuracy of saccadic eye movements revealed a main effect of task condition $[F(1,19)=14.43, p=.001]$. Participants were slightly more accurate during the VS task compared to the MS task. Indeed, the average absolute errors were $.3^{\circ}$ and $.6^{\circ}$, respectively, for the VS and MS tasks. These results are consistent with previous findings regarding the characteristics of saccades to remembered targets (Ohtsuka et al., 1989). Neither the main effect of memorized/ cued side nor the interaction between task condition and memorized/ cued side reached significance.

\section{ERP data}

Analysis of the ERP amplitudes revealed the time course of the differential activation of cortical areas between MS and VS task conditions. As expected, appearance of the visual cue yielded ERP waveforms with a similar general pattern in both conditions, since the two task paradigms were very similar. Therefore, significant differences between the conditions could be ascribed to mechanisms related to the processing and maintenance of the spatial location in the MS task (see Fig. 2). Repeated-measures ANOVAs comparing mean amplitudes in six different time windows were used to test for the effects of task condition (MS, VS), hemisphere (ipsilateral, contralateral relative to the memorized/cued side) and electrode site. The analyzed intervals are highlighted by boxes in Fig. 2 .

(a) The first difference in the ERP between task conditions (Fig. 2, Box a) was in the visual potential N1 during the time between 160 and $180 \mathrm{~ms}$. The MS condition generated a significantly larger N1 in comparison to the VS condition over all of the parietal-occipital electrodes, as revealed by the main effect of task condition $[F(1$, $19)=8.06, p=.01]$. The main effect of electrode sites $[F(3,57)=$ $3.93, p=.02$ ] showed that, in both conditions, the $\mathrm{N} 1$ component reached the largest amplitude in PO7/8. Neither the main effect of hemisphere nor interactions between the factors were significant, showing that $\mathrm{N} 1$ was not significantly lateralized.

(b) Immediately after the N1 component, a large difference in the ERPs of the MS and VS conditions was observed in the most

$\mathrm{FP} 1 / 2 \mathrm{co}$
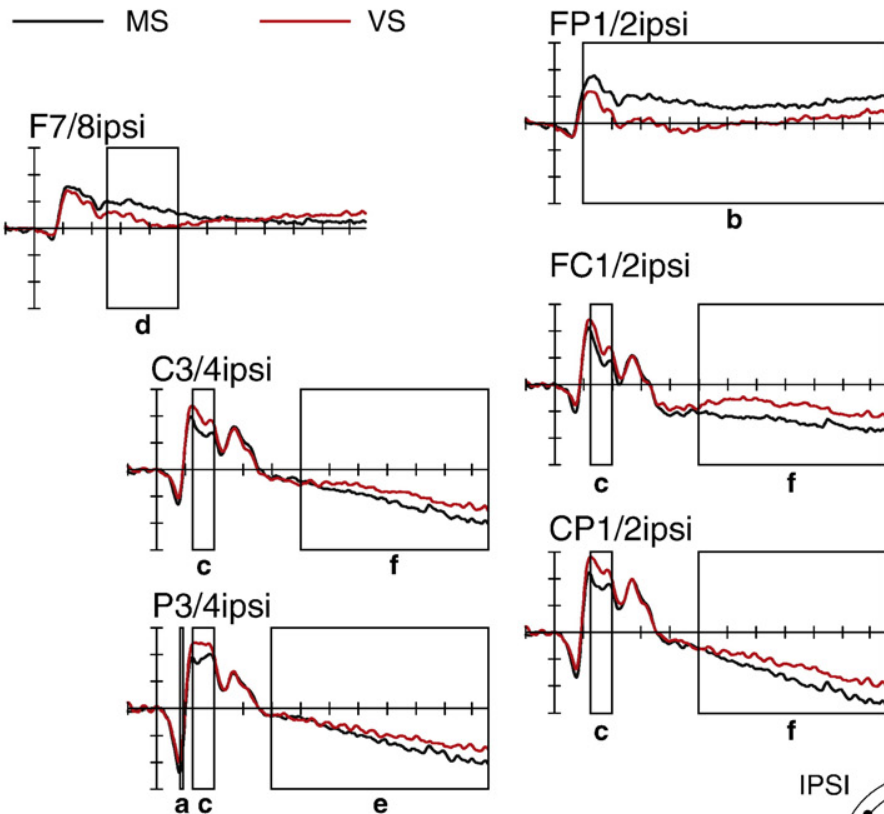

FC1/2ipsi

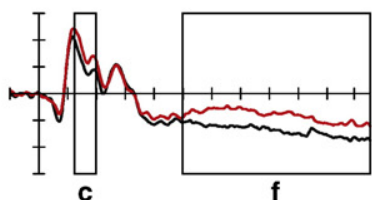

CP1/2ipsi
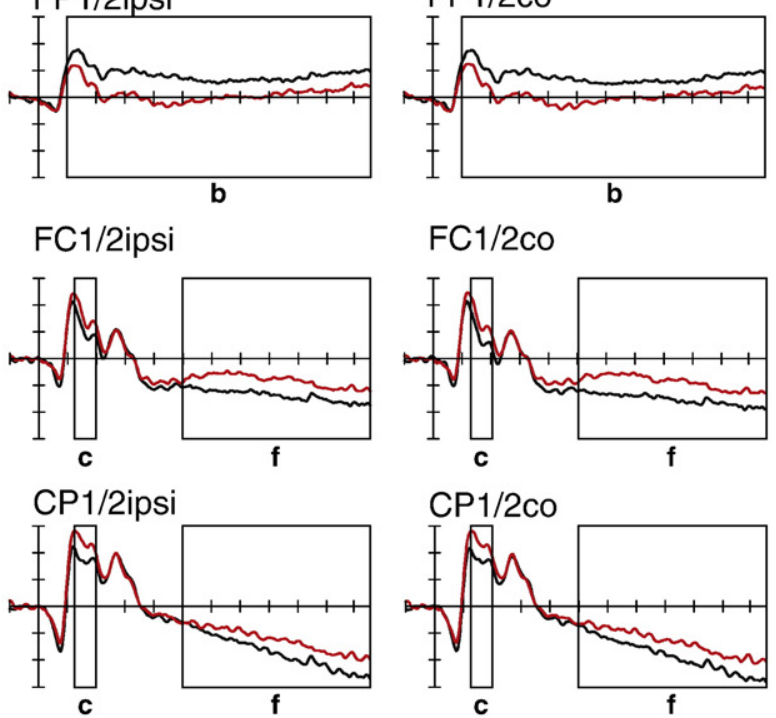

$\mathrm{FC} 1 / 2 \mathrm{co}$

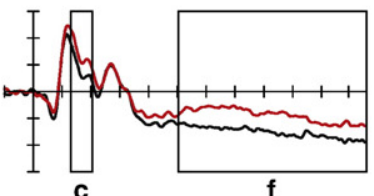

$\mathrm{CP} 1 / 2 \mathrm{Co}$
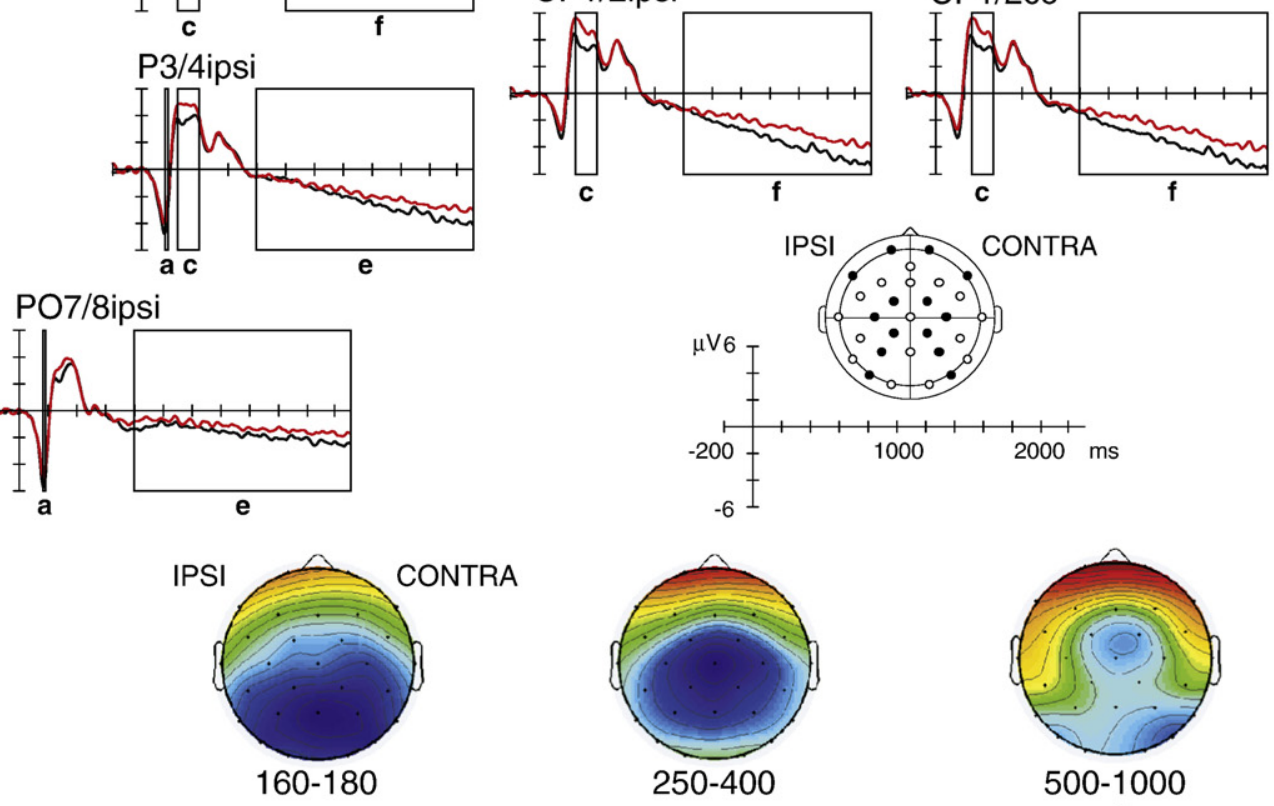

a

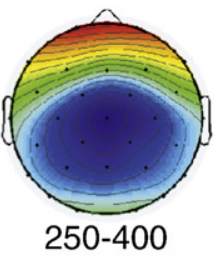

C

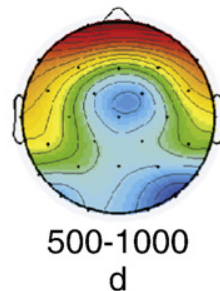

d
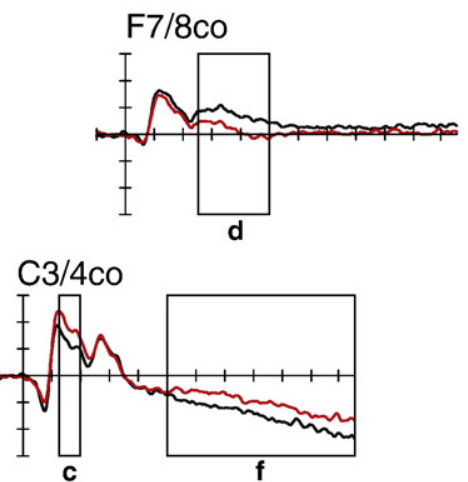

$\mathrm{P} 3 / 4 \mathrm{Co}$
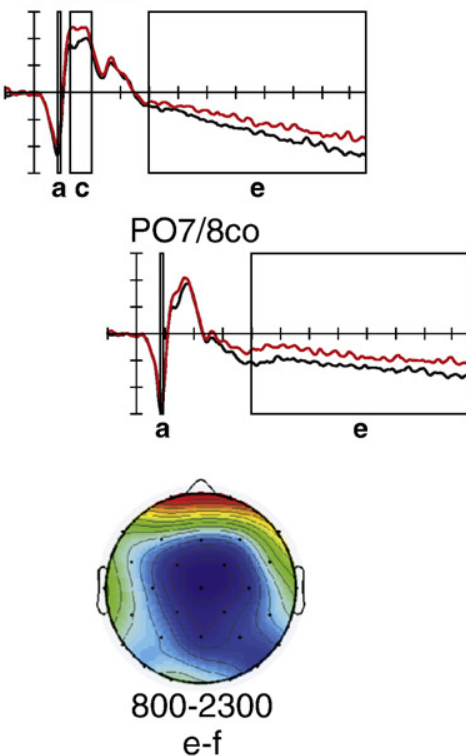

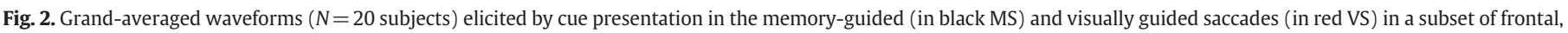

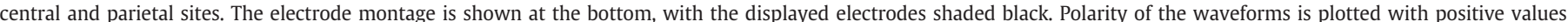

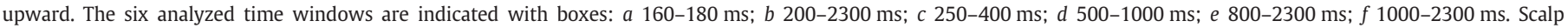

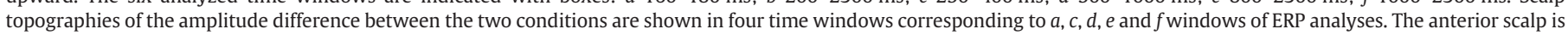
shown on the top and the contralateral scalp on the right side. Contralateral and ipsilateral refer to the memorized/cued side. 
anterior part of the frontal region $[F(1,19)=9.33, p=.006]$, as can be seen in Fig. 2, Box b. This difference was present until the end of the delay-phase (200-2300 ms). Specifically, we measured a more positive waveform from the FP1/2 electrodes during the MS task in comparison to the VS task; this difference was equivalent in both the ipsilateral and contralateral hemispheres. Indeed, neither the main effect of hemisphere nor the interactions between factors reached significance.

(c) After the N1 component, a difference in the ERPs between the MS and VS tasks was measured from the central-parietal electrodes ( $\mathrm{FC} 1 / 2, \mathrm{C} 3 / 4, \mathrm{CP} 1 / 2, \mathrm{CP} 5 / 6, \mathrm{P} 3 / 4)$ for a limited time window (i.e., $250-400 \mathrm{~ms}$ ), as indicated by the main effect of task condition $[F(1,19)=14.57, p=.001]$ (Fig. 2, Box c). This difference coincided with two positive deflections, which showed a smaller amplitude in the MS as compared to the VS condition. An interaction of the hemisphere factor with the electrode site $[F(4,76)=6.35, p<.001]$ was found, such that, over the two central electrodes (i.e., FC1/2, C3/4), the ERPs were more negative in the hemisphere contralateral to the memorized/cued side as compared to the ipsilateral hemisphere. This lateralized effect was present in both conditions, but it was accentuated in the MS task, as revealed by the three way interaction task condition $\times$ hemisphere $\times$ electrode site $[F(4,76)=2.48, p=.05]$.

(d) In the subsequent time window (500-1000 ms), the MS task elicited a larger positive slow deflection in comparison to the VS condition in the most lateral frontal-temporal electrodes $(\mathrm{F} 7 / 8, \mathrm{~T} 7 / 8, \mathrm{FC} 5 / 6)$ [task condition: $F(1,19)=7.35, p=.01$ ] (Fig. 2, Box d). This difference was localized specifically in F7/8, as suggested by the interaction task condition $\times$ electrode site $[F$ $(2,38)=6.77, p=.007]$. The main effect hemisphere $[F(1,19)=$ $8.54, p=.009]$ indicated that there was a lateralized difference in both task conditions, with ERPs in the hemisphere contralateral to the memorized/cued side being more negative than those in the ipsilateral hemisphere.

(e) Starting at $800 \mathrm{~ms}$ after the onset of the cue, a sustained negative slow wave resembling the CDA developed throughout the parietal electrodes $(\mathrm{P} 3 / 4, \mathrm{P} 7 / 8, \mathrm{PO} / 8)$, where it persisted throughout the duration of the memory/delay-phase in both task conditions (i.e., 800-2300 ms) (Fig. 2, Box e). In both conditions, the CDA showed a larger amplitude when measured from the hemisphere contralateral to the memorized/ cued side [hemisphere: $F(1,19)=21.39, p<.001$ ] (Fig. 3). This decreasing voltage reached a larger amplitude in the MS task in comparison to the VS task [task condition: $F(1,19)=4.73$, $p=.04]$, particularly in the hemisphere contralateral to the memorized/cued side, as revealed by the interaction task condition $\times$ hemisphere $[F(1,19)=6.71, p=.02]$.

(f) Finally, a sustained negative slow wave was detected over the central electrodes (FC1/2, C3/4, CP1/2), where it began to develop just $200 \mathrm{~ms}$ after the parietal CDA and lasted until the end of the memory/delay-phase (i.e., 1000-2300 ms) (Fig. 2, Box f). This component exhibited characteristics similar to the parietal CDA: it was larger in the MS task than in the VS task [task condition: $F(1,19)=7.76, p=.01$ ] and, in both conditions, it was larger in the hemisphere contralateral to the memorized/cued side [hemisphere: $F(1,19)=21.39, p<.001]$, specifically in the $\mathrm{C} 3 / 4$ electrodes [hemisphere $\times$ electrode site: $F(1$, $19)=21.39, p<.001]$. The factor of task condition, however, did not interact with hemisphere or electrode site.

\section{Cortical localization data}

The analysis performed with sLORETA provided information about differential activation between MS and VS task conditions in five ROIs (see the Cortical localization analysis section in Materials and

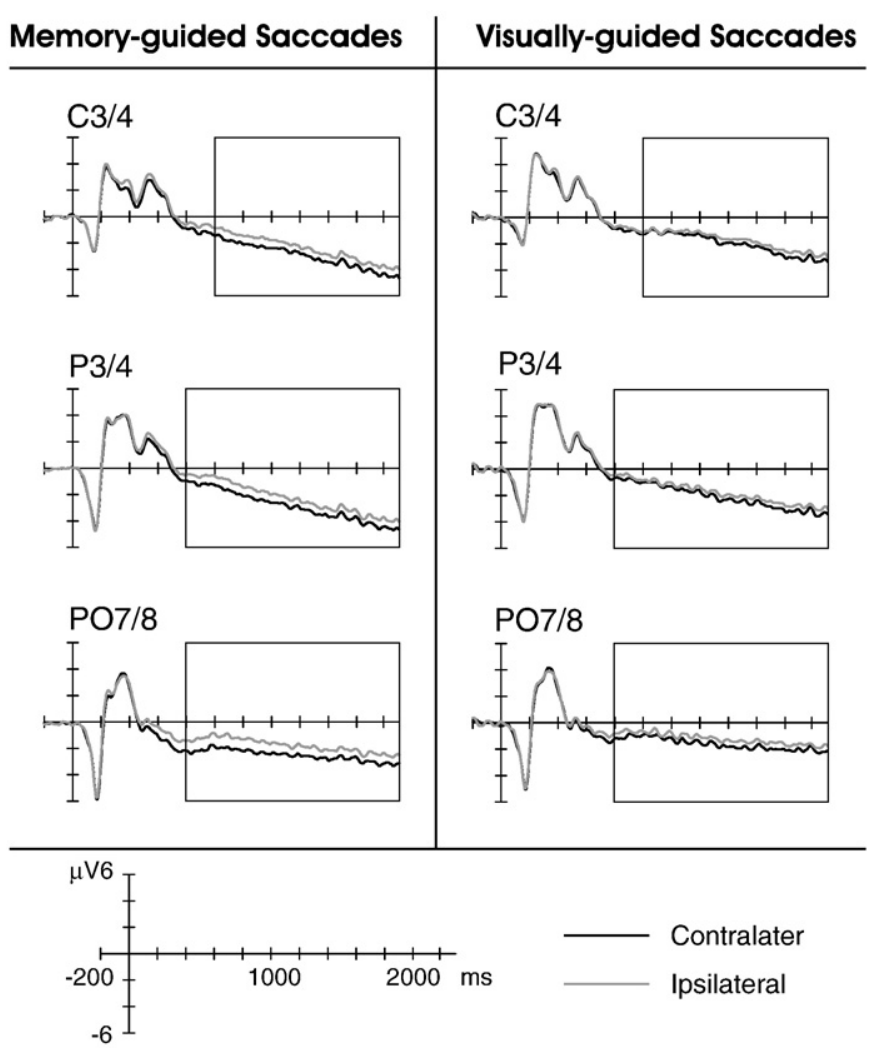

Fig. 3. Contralateral (in black) and ipsilateral (in gray) activity at central and posterior electrodes elicited by the cue presentation in the memory-guided (on the left) and visually guided (on the right) saccade tasks. In both the conditions a larger amplitude was recorded over the hemisphere contralateral to the memorized/cued side, and this ipsi/contralateral difference was higher in the memory-guided task.

methods), and allowed us to characterize the time course of these differences. Two-way ANOVAs, with task condition (MS, VS) and hemisphere (ipsilateral, contralateral relative to the memorized/cued side) as factors, were applied at each time point for each ROI. The results are presented in Fig. 4. These analyses led to the following results:

1) The ACC was significantly more activated in the MS than in the VS condition from $300 \mathrm{~ms}$ after the cue onset to the end of the epoch, as suggested by the main effect of task condition [all $F$ values $(1,19)>4.39, p$ values $<.05]$. No significant differences due to the main effect of hemisphere or to the interaction between factors were observed.

2) The dlPFC was more active in the MS than in the VS condition from 700 to $960 \mathrm{~ms}$ and from 1030 to $1060 \mathrm{~ms}$ [task condition: all $F$ values $(1,19)>4.38, p$ values $<.05]$. The main effect of hemisphere was also significant in a short interval between 2100 and $2120 \mathrm{~ms}$ [all $F$ values $(1,19)>4.06, p$ values $<.05$ ], showing more activation in the ipsilateral than in the contralateral hemisphere. No significant interaction between task condition and hemisphere was obtained.

3) The FEFs were more active in the MS than in the VS condition at 160 to $180 \mathrm{~ms}$ and at 290 to $1060 \mathrm{~ms}$ [task condition: all $F$ values $(1,19)>4.53, p$ values $<.05]$. Neither the main effect of hemisphere nor any interaction between the factors reached significance.

4) The IPL was more activated in the MS than in the VS condition for a persistent interval from $330 \mathrm{~ms}$ to the end of the epoch [task condition: all $F$ values $(1,19)>4.38, p$ values $<.05]$. The main effect of hemisphere was also significant [all $F$ values $(1,19)>4.41$, $p$ values $<.05$ ], revealing a larger activation in the ipsilateral than in the contralateral hemisphere from 2050 to $2240 \mathrm{~ms}$. 


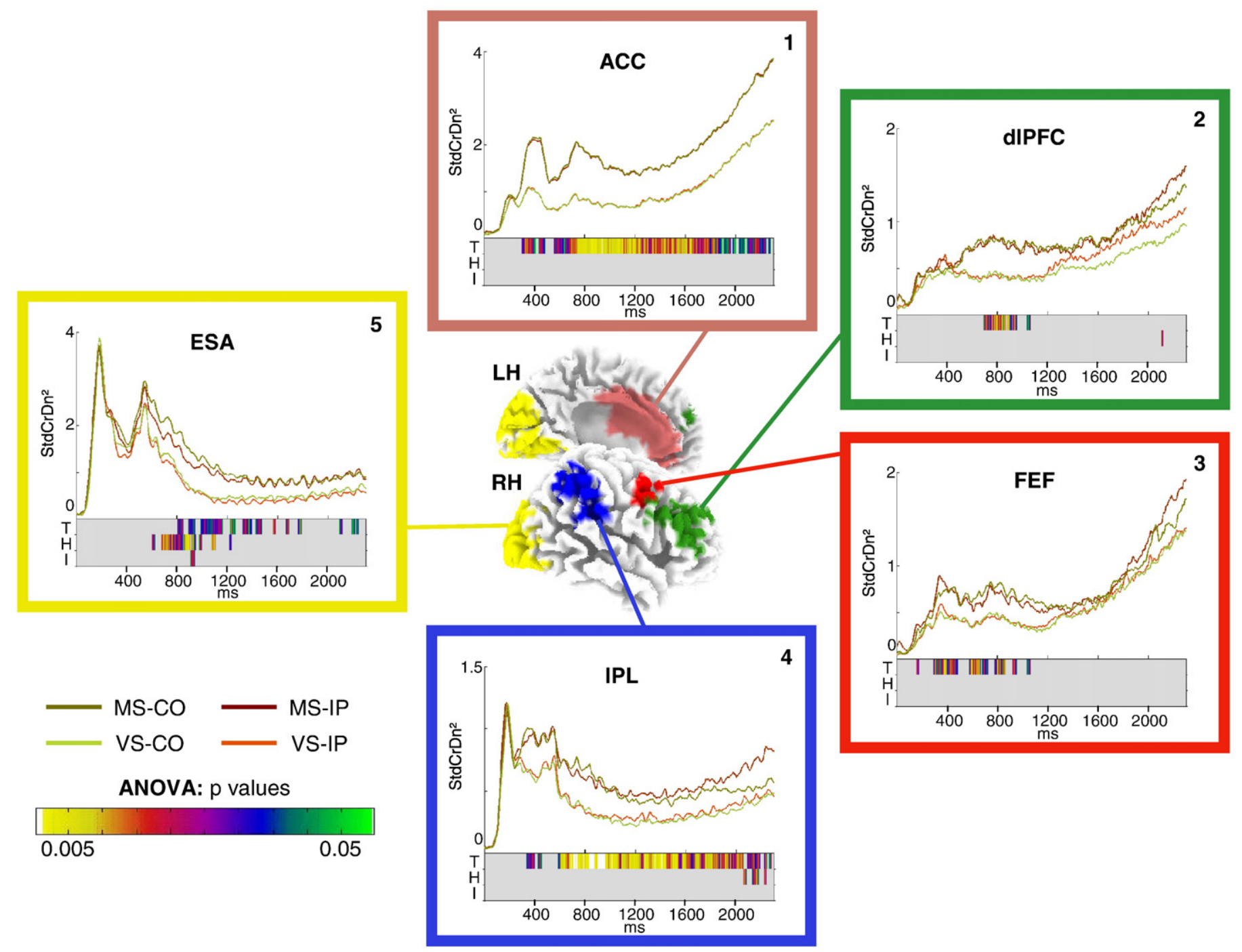

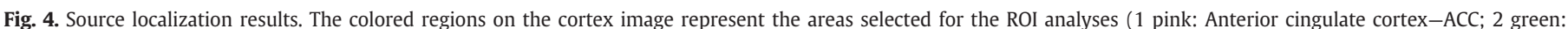

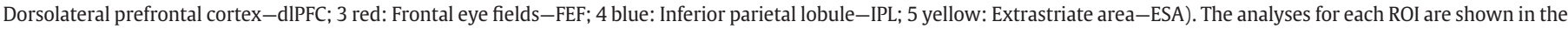

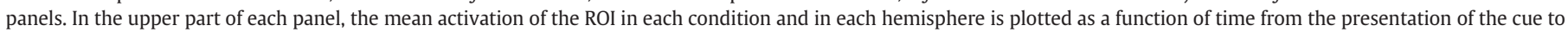

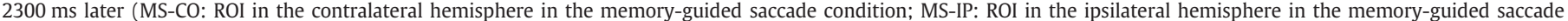

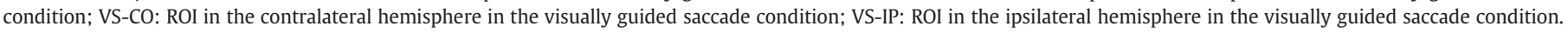

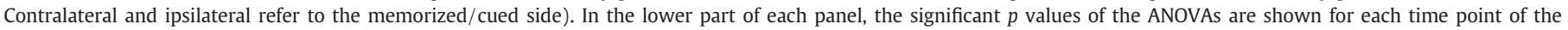
epoch (T: main effect task condition; H: main effect hemisphere i.e., ipsilateral, contralateral relative to the memorized/cued side; I: interaction task condition $\times$ hemisphere).

5) Extrastriate areas showed a main effect of task condition [all $F$ values $(1,19)>4.38, p$ values $<.05]$, which showed a higher activation in the MS than in the VS condition throughout the entire 800 to $1470 \mathrm{~ms}$ time window and sporadically up to $2230 \mathrm{~ms}$ after the cue presentation. From 600 to $1230 \mathrm{~ms}$, the contralateral hemisphere was more activated than the ipsilateral one in both conditions [hemisphere: all $F$ values $(1,19)>4.40, p$ values $<.05$ ]. However in the time window between 910 and $940 \mathrm{~ms}$, this difference was larger in the MS than in the VS condition, as suggested by the task condition $\times$ hemisphere interaction [all $F$ values $(1,19)>4.76$, $p$ values $<.05$ ].

\section{Discussion}

The aim of the present study was to investigate the mechanisms underlying the maintenance of spatial WM. To this end, we compared ERPs elicited by an MS and a VS task, and applied a brain tomography localization method to track the timing and the neural sources of the cortical activity with a paradigm built to eliminate possible confounds.
The two task conditions were carefully designed to control for the visual properties of the stimuli and for cognitive processes not specifically related to WM (e.g., arousal, sustained attention, general response preparation, etc.). In particular, subjects were induced to shift spatial attention and to prepare a saccadic response towards a visual hemifield in both the conditions. However, in the MS condition, subjects were required to memorize the precise location of the cue and to execute an MS towards it at the end of the delay-phase. In the VS task, instead, subjects were unaware of the amplitude of the saccade to be executed at the end of the delay-phase, such that they were unable to prepare a specific motor plan.

Comparison of ERPs measured in MS and VS tasks revealed two sustained differences during the delay-phase. One difference in the ERP was localized in the most anterior part of the frontal region and developed very early after cue presentation (i.e., $200 \mathrm{~ms}$ ). Considering its early onset, this effect is unlikely to indicate the maintenance of information. It has been previously ascribed to active inhibition of oculomotor responses towards the cued location during the delayphase (Evdokimidis et al., 2001; Rama et al., 1995). In the present study, 
precise cortical localization analyses revealed that ACC exhibited a sustained differential activation between conditions throughout the entire delay-phase, suggesting that this region is a likely source for this ERP effect. ACC is considered part of the fronto-parietal attention network (Posner and Dehaene, 1994), and is thought to be involved in attentive control and monitoring for conflict during stimulus processing and response selection (Cohen et al., 2000; Luks et al., 2002; Petit et al., 1998). There is also evidence that ACC may be involved in the attentional control process at early stages following preparatory cues prior to target presentation (Gitelman et al., 1999). Accordingly, it is possible that ACC exerted attentive control over the regions that maintained the precise spatial location or, alternatively, ACC monitored saccade inhibition toward the memorized location.

The second sustained difference in the ERPs measured in the MS and VS conditions arose later (i.e., $800 \mathrm{~ms}$ ) and was observed for surface data in a wider cortical region (including central, parietal and occipital areas) as a large negative slow wave. This ERP potential resembles the CDA, considered an electrophysiological correlate of visual WM (Klaver et al., 1999; McCollough et al., 2007; Vogel and Machizawa, 2004). In the present study, a sustained difference in the amplitude of the CDA between the two task conditions was found over the parietal, occipital and central electrode sites. This cortical distribution suggests that, not only the posterior perceptual cortical regions, but also more anterior motor areas may be involved in the maintenance of spatial information. Nevertheless cortical localization analyses revealed a sustained differential activation between MS and VS tasks for the entire delay-phase in the IPL and extrastriate areas, but not in the central regions. The FEFs were differentially activated to a greater extent in the MS than in the VS condition only during the first part of the delay-phase. Subsequently, FEF activation began to increase in both conditions, eventually reaching the same level of current density and canceling out the previous difference. This increasing activation seems to reflect activity related to a general motor preparation or to a larger attentional involvement for the impending eye movements, since the subjects could make an accurate prediction of when the future saccadic response would occur in both MS and VS tasks. The FEFs have a well established role both in the voluntary control of saccadic eye movements (Bruce and Goldberg, 1985; Bruce et al., 1985) and in covert spatial attention (Armstrong et al., 2006; Moore and Armstrong, 2003; Moore and Fallah, 2001), while their precise function in spatial working memory is still debated. The pattern of activity here observed appears consistent with a system in which during the active maintenance of spatial information FEFs bias, via attention, the same regions that initially respond during visual encoding of the information (Awh et al., 2000; Postle, 2006). In line with this conclusion is a recent study in monkeys by Armstrong et al. (2009). They found a persistent activity in FEF neurons during a working memory task although the task did not involve saccades. The sustained activation of the FEF, which might not be related to the maintenance of an oculomotor plan, was ascribed to a sustained attention toward the visual processing at remembered locations.

The timing and localization of activity within the IPL and extrastriate areas reveal that these regions participate in both initial sensory processing of visual cues and in the short-term maintenance of spatial location. In agreement with the key role of attentional rehearsal in spatial maintenance (Awh et al., 2000; Awh and Jonides, 2001; Awh et al., 1999; Postle et al., 2004), the topographically organized extrastriate areas were specifically modulated according to the cued side. Indeed, both in the ERP (i.e., CDA component) and in the localization analyses, the contralateral hemisphere was more activated than the ipsilateral one. Although posterior parietal cortex also tends to represent contralateral space (Schluppeck et al., 2005; Sereno et al., 2001), the current density of the IPL showed no consistent differences between hemispheres. However, lateralized activity during spatial WM tasks has not always been reported (Curtis and D'Esposito, 2006; Medendorp et al., 2006; Schluppeck et al., 2006), probably due to the large power needed to detect such small differences (Srimal and Curtis, 2008).

The rational for the interpretation of these results is that a brain region is believed to play a critical role in maintenance of spatial information only when its activity persists throughout the entire delay-phase between the sensory-phase and the motor-phase. Whether this maintained activity reflects a retrospective representation of the sensory information or encodes a prospective plan for the motor response is a matter of debate (Curtis et al., 2004; Quintana and Fuster, 1999; Srimal and Curtis, 2008).

We would have expected a larger sustained activity of FEF in MS than in VS task condition during all the delay-phase, if FEF had played a relevant role in the storage of spatial information. On the contrary, although the precise oculomotor coordinates for the saccadic response were provided before the delay-phase only in MS condition, the FEFs showed a differential greater sustained activity in MS condition only in the first part of the delay-phase, while in the second part FEF activation was high in both conditions. Notably this differential sustained activity was instead present over IPL. Thus, our data are inconsistent with the hypothesis that the FEFs play a relevant role in the storage of spatial information by maintaining the saccadic plan necessary to acquire the target location. This discrepancy with literature may depend on the task designs used in the previous studies in which the protocols probably did not completely control for processes not specifically related to WM, such as the general response preparation. In the present study, timing of the activation of the FEFs might suggest that they participate in the transformation of spatial information into a motor coordinate framework. Following the differences in the N1 potential between tasks, which reflect the heavier perceptual coding required by the spatial WM condition itself, a difference in the ERPs measured in the MS and VS tasks was found in the central-parietal electrodes for a limited time window. Although it was difficult to recognize clearly labeled ERP components, the timing and the topography of this effect suggests that it reflects activity related to visuo-motor transformation. In the same temporal interval (about $400 \mathrm{~ms}$ ), the cortical localization analyses revealed differential activation of both the FEF and the IPL between conditions. These areas, which are highly interconnected and send and receive projections to and from almost identical cortical and subcortical areas, are both presumed to play important roles in the representation of space and in the transformation of spatial information into a motor coordinate framework (Andersen and Buneo, 2002; Bruce and Goldberg, 1985; Chafee and Goldman-Rakic, 1998; Culham and Valyear, 2006). Therefore, following perceptual coding of the cue, which depends on the occipitoparietal areas, both the IPL and the FEF may have contributed to the conversion from visuo-spatial to motor information, process which was more demanding in MS than in VS, accounting for the different activation found between the conditions in this phase.

Following the visuo-motor effect, a restricted difference in the ERPs measured in the MS and VS conditions was observed over the lateral frontal areas. Interestingly, this effect was concurrent with a brief differential activation of the dIPFC as revealed by sLORETA analyses. There is ample evidence indicating that the dIPFC plays an important role in WM, although its involvement in the storage of information is a highly controversial issue (Postle, 2006). Considering the timing of dIPFC activation, these findings suggest that dIPFC is more likely involved in executive control functions, such as selection of the appropriate memory-guided response as proposed by several groups (Curtis et al., 2004; Owen et al., 1996; Pochon et al., 2001; Rowe et al., 2000).

On the whole, these data suggest that the spatial location of the saccadic target is exclusively maintained in the IPL and in the extrastriate areas. However, given the novelty of the methodology used in the present study, further investigations will be necessary to confirm these results. Although the use of SLORETA allowed to overcome the spatial limitations of the ERP method, it cannot provide the same precision of functional imaging methods. In addition, the 
possibility remains that activity seen within an ROI stems from other extra-ROI sources. Nevertheless, the present results are plausible. The posterior parietal cortex may participate in maintenance of a motor plan, even though the FEFs are the principal candidate to be involved when subjects are biased towards the use of a prospective motor code, like in this task (i.e., when the precise metrics of the MS is known throughout the delay-phase) (Curtis, 2006; Curtis et al., 2004). Recent studies have provided clear evidence indicating that the human posterior parietal cortex exhibits some of the response properties that characterize the monkey lateral intraparietal area (Fink, 2005; Schluppeck et al., 2006). Posterior parietal cortex has been shown to play an important role, not only in perception and attention allocation, but also in the planning and production of saccadic eye movements. Indeed, this area responds while computing the sensorymotor transformation involved in making a movement to a stimulus and while maintaining the intention to make such a movement (for review, see Andersen and Buneo, 2002; Glimcher, 2003; Goldberg et al., 2002). At theoretic level, the data of the present study are in agreement also with recent evidence (for a review, see Postle, 2006) that debate the timeliness of considering prospective and retrospective codes as two separate mechanisms for the retention of location information; in fact, these "mechanisms" appear to be more easily explained as two points along a single sensorimotor continuum.

In conclusion, by exploiting techniques that provide exquisite temporal resolution and reasonably precise anatomical localization, this study provides evidence supporting the key role of the IPL in the storage of spatial information during a WM guided saccade, even when subjects are biased towards the use of a prospective motor code.

\section{Acknowledgments}

We thank Mattia Marangon and Katiuscia Sosta for help with the recording. This work was supported by a grant from the AFaR and from the Ministero dell'Università e della Ricerca PRIN 2007.

\section{References}

AEEGS, 1991. American Electroencephalographic Society guidelines for standard electrode position nomenclature. J. Clin. Neurophysiol. 8, 200-202.

Andersen, R.A., Buneo, C.A., 2002. Intentional maps in posterior parietal cortex. Annu. Rev. Neurosci. 25, 189-220.

Armstrong, K.M., Fitzgerald, J.K., Moore, T., 2006. Changes in visual receptive fields with microstimulation of frontal cortex. Neuron 50, 791-798.

Armstrong, K.M., Chang, M.H., Moore, T., 2009. Selection and maintenance of spatia information by frontal eye field neurons. J. Neurosci. 29, 15621-15629.

Awh, E., Jonides, J., 2001. Overlapping mechanisms of attention and spatial working memory. Trends Cogn. Sci. 5, 119-126.

Awh, E., Jonides, J., Smith, E.E., Buxton, R.B., Frank, L.R., Love, T., Wong, E.C., Gmeindl, L, 1999. Rehearsal in spatial working memory: evidence from neuroimaging. Psychol. Sci. 10, 433-437.

Awh, E., Anllo-Vento, L., Hillyard, S.A., 2000. The role of spatial selective attention in working memory for locations: evidence from event-related potentials. J. Cogn. Neurosci. 12, 840-847.

Baddeley, A.D., 1986. Working Memory. Oxford Univ. Press, Oxford.

Brignani, D., Maioli, C., Maria Rossini, P., Miniussi, C., 2007. Event-related power modulations of brain activity preceding visually guided saccades. Brain Res. 1136, 122-131.

Bruce, C.J., Goldberg, M.E., 1985. Primate frontal eye fields: I. Single neurons discharging before saccades. J. Neurophysiol. 53, 603-635.

Bruce, C.J., Goldberg, M.E., Bushnell, M.C., Stanton, G.B., 1985. Primate frontal eye fields II. Physiological and anatomical correlates of electrically evoked eye movements. J. Neurophysiol. 54, 714-734.

Chafee, M.V., Goldman-Rakic, P.S., 1998. Matching patterns of activity in primate prefrontal area $8 \mathrm{a}$ and parietal area 7ip neurons during a spatial working memory task. J. Neurophysiol. 79, 2919-2940.

Cohen, J.D., Botvinick, M., Carter, C.S., 2000. Anterior cingulate and prefrontal cortex: who's in control? Nat. Neurosci. 3, 421-423.

Culham, J.C., Valyear, K.F., 2006. Human parietal cortex in action. Curr. Opin. Neurobiol. $16,205-212$.

Curtis, C.E., 2006. Prefrontal and parietal contributions to spatial working memory. Neuroscience 139, 173-180.

Curtis, C.E., D'Esposito, M., 2003. Persistent activity in the prefrontal cortex during working memory. Trends Cogn. Sci. 7, 415-423.
Curtis, C.E., D'Esposito, M., 2006. Selection and maintenance of saccade goals in the human frontal eye fields. J. Neurophysiol. 95, 3923-3927.

Curtis, C.E., Rao, V.Y., D'Esposito, M., 2004. Maintenance of spatial and motor codes during oculomotor delayed response tasks. J. Neurosci. 24, 3944-3952.

D'Esposito, M., 2007. From cognitive to neural models of working memory. Philos. Trans. R. Soc. Lond. B Biol. Sci. 362, 761-772.

Evdokimidis, I., Smyrnis, N., Constantinidis, T.S., Gourtzelidis, P., Papageorgiou, C., 2001. Frontal-parietal activation differences observed before the execution of remembered saccades: an event-related potentials study. Brain Res. Cogn Brain Res. 12, $89-99$.

Fink, G., 2005. The functional organization of the intraparietal sulcus in humans and monkeys. J. Anat. 207, 3-17.

Fuchs, M., Kastner, J., Wagner, M., Hawes, S., Ebersole, J.S., 2002. A standardized boundary element method volume conductor model. Clin. Neurophysiol. 113, 702-712.

Fuster, J.M., 1995. Memory in the Cerebral Cortex. MIT Press, Cambridge, MA.

Gitelman, D.R., Nobre, A.C., Parrish, T.B., LaBar, K.S., Kim, Y.H., Meyer, J.R., Mesulam, M., 1999. A large-scale distributed network for covert spatial attention: further anatomical delineation based on stringent behavioural and cognitive controls. Brain 122, 1093-1106.

Glimcher, P.W., 2003. The neurobiology of visual-saccadic decision making. Annu. Rev. Neurosci. 26, 133-179.

Goldberg, M.E., Bisley, J., Powell, K.D., Gottlieb, J., Kusunoki, M., 2002. The role of the lateral intraparietal area of the monkey in the generation of saccades and visuospatial attention. Ann. N. Y. Acad. Sci. 956, 205-215.

Guthrie, D., Buchwald, J.S., 1991. Significance testing of difference potentials. Psychophysiology 28, 240-244.

Hikosaka, O., Wurtz, R.H., 1983. Visual and oculomotor functions of monkey substantia nigra pars reticulata: III. Memory-contingent visual and saccade responses. J. Neurophysiol. 49, 1268-1284.

Hillyard, S.A., Picton, T.W., 1979. Event-related brain potentials and selective information processing in man. In: Desmedt, J.E. (Ed.), Progress in Clinical Neurophysiology. : Cognitive Components in Cerebral Event-Related Potentials and Selective Attention. Karger, Basel, pp. 1-50.

Klaver, P., Talsma, D., Wijers, A.A., Heinze, H.J., Mulder, G., 1999. An event-related brain potential correlate of visual short-term memory. Neuroreport 10, 2001-2005.

Lancaster, J.L., Woldorff, M.G., Parsons, L.M., Liotti, M., Freitas, C.S., Rainey, L., Kochunov, P.V., Nickerson, D., Mikiten, S.A., Fox, P.T., 2000. Automated Talairach atlas labels for functional brain mapping. Hum. Brain Mapp. 10, 120-131.

Luks, T.L., Simpson, G.V., Feiwell, R.J., Miller, W.L., 2002. Evidence for anterior cingulate cortex involvement in monitoring preparatory attentional set. Neuroimage 17, 792-802.

McCollough, A.W., Machizawa, M.G., Vogel, E.K., 2007. Electrophysiological measures of maintaining representations in visual working memory. Cortex 43, 77-94.

Medendorp, W.P., Goltz, H.C., Vilis, T., 2006. Directional selectivity of BOLD activity in human posterior parietal cortex for memory-guided double-step saccades. J. Neurophysiol. 95, 1645-1655.

Moore, T., Armstrong, K.M., 2003. Selective gating of visual signals by microstimulation of frontal cortex. Nature 421, 370-373.

Moore, T., Fallah, M., 2001. Control of eye movements and spatial attention. Proc. Natl. Acad. Sci. U. S. A. 98, 1273-1276.

Murray, M.M., Wylie, G.R., Higgins, B.A., Javitt, D.C., Schroeder, C.E., Foxe, J.J., 2002. The spatiotemporal dynamics of illusory contour processing: combined high-density electrical mapping, source analysis, and functional magnetic resonance imaging. J. Neurosci. 22, 5055-5073.

Nobre, A., Correa, A., Coull, J., 2007. The hazards of time. Curr. Opin. Neurobiol. 17, $465-470$

Ohtsuka, K., Sawa, M., Takeda, M., 1989. Accuracy of memory-guided saccades. Ophthalmologica $198,53-56$.

Oldfield, R.C., 1971. The assessment and analysis of handedness: the Edinburgh inventory. Neuropsychologia 9, 97-113.

Owen, A.M., Evans, A.C., Petrides, M., 1996. Evidence for a two-stage model of spatial working memory processing within the lateral frontal cortex: a positron emission tomography study. Cereb. Cortex 6, 31-38.

Pascual-Marqui, R.D., 2002. Standardized low-resolution brain electromagnetic tomography (sLORETA): technical details. Methods Find. Exp. Clin. Pharmacol. 24 (Suppl D), 5-12.

Pascual-Marqui, R.D., 2007. Discrete, 3D distributed, linear imaging methods of electric neuronal activity: Part 1. Exact, zero error localization. arXiv:07103341 [math-ph].

Paus, T., 1996. Location and function of the human frontal eye-field: a selective review. Neuropsychologia 34, 475-483.

Petit, L., Courtney, S.M., Ungerleider, L.G., Haxby, J.V., 1998. Sustained activity in the medial wall during working memory delays. J. Neurosci. 18, 9429-9437.

Pochon, J.B., Levy, R., Poline, J.B., Crozier, S., Lehericy, S., Pillon, B., Deweer, B., Le Bihan, D., Dubois, B., 2001. The role of dorsolateral prefrontal cortex in the preparation of forthcoming actions: an fMRI study. Cereb. Cortex 11, 260-266.

Posner, M.I., Dehaene, S., 1994. Attentional networks. Trends Neurosci. 17, 75-79.

Postle, B.R., 2006. Working memory as an emergent property of the mind and brain. Neuroscience 139, 23-38.

Postle, B.R., D'Esposito, M., 2003. Spatial working memory activity of the caudate nucleus is sensitive to frame of reference. Cogn. Affect. Behav. Neurosci. 3, 133-144.

Postle, B.R., Awh, E., Jonides, J., Smith, E.E., D'Esposito, M., 2004. The where and how of attention-based rehearsal in spatial working memory. Brain Res. Cogn. Brain Res. 20, 194-205. 
Quintana, J., Fuster, J.M., 1999. From perception to action: temporal integrative functions of prefrontal and parietal neurons. Cereb. Cortex 9, 213-221.

Rama, P., Carlson, S., Kekoni, J., Hamalainen, H., 1995. A spatial oculomotor memorytask performance produces a task-related slow shift in human electroencephalography. Electroencephalogr. Clin. Neurophysiol. 94, 371-380.

Rowe, J.B., Toni, I., Josephs, O., Frackowiak, R.S., Passingham, R.E., 2000. The prefrontal cortex: response selection or maintenance within working memory? Science $288,1656-1660$.

Schluppeck, D., Glimcher, P., Heeger, D.J., 2005. Topographic organization for delayed saccades in human posterior parietal cortex. J. Neurophysiol. 94, 1372-1384.
Schluppeck, D., Curtis, C.E., Glimcher, P.W., Heeger, D.J., 2006. Sustained activity in topographic areas of human posterior parietal cortex during memory-guided saccades. J. Neurosci. 26, 5098-5108.

Sereno, M.I., Pitzalis, S., Martinez, A., 2001. Mapping of contralateral space in retinotopic coordinates by a parietal cortical area in humans. Science 294, 1350-1354.

Srimal, R., Curtis, C.E., 2008. Persistent neural activity during the maintenance of spatial position in working memory. Neuroimage $39,455-468$.

Vogel, E.K., Machizawa, M.G., 2004. Neural activity predicts individual differences in visual working memory capacity. Nature $428,748-751$. 